\title{
A FAST SPECTRUM SENSING FOR CP-OFDM Cognitive Radio BASEd ON AdAPTIVE THRESHOLDING
}

\author{
Kamel Berbra $^{\mathrm{a}, *}$, Mourad Barkat ${ }^{\mathrm{b}}$, Fulvio Gini ${ }^{\mathrm{c}}$, Maria Greco, \\ and Pietro Stinco ${ }^{\mathrm{c}}$
}

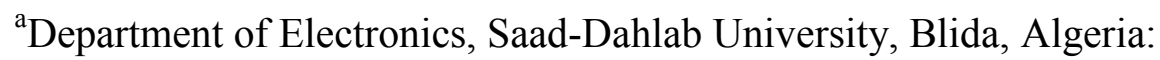

berbra.kamel@gmail.com

${ }^{\mathrm{b}}$ Department of Electrical and Computer Engineering Technology, Valencia College, Orlando, Florida, 32811: mbarkat58@gmail.com

${ }^{c}$ Department of “Ingeneria dell’Informazione”, Pisa University, Pisa, Italy: f.gini@ing.unipi.it

*Corresponding Author 
ABSTRACT-Recently, cyclostationarity (CS) based detection methods exploiting the autocorrelation periodicity property of the orthogonal frequency division multiplexing (OFDM) signals attracted a lot of attention. These detection methods are more complex than energy detection but they have better detection performance in low-SNR regimes. The drawback, however, is their extensive computational complexity. In this paper, we propose a computationally efficient spectrum sensing method for detecting unsynchronized OFDM signals in additive white Gaussian noise (AWGN). The proposed method exploits the second-order CS property of OFDM signals to set an adaptive threshold, which achieves the desired constant false alarm rate (CFAR) property. The complexity of the proposed method is significantly reduced compared with the scheme proposed in [22]. Monte-Carlo simulations illustrate that the performance of the proposed detector outperforms the classical energy detector (ED) and the other existing spectrum sensing algorithms in the open literature in addition to guaranteeing a low sensing time.

Keywords - Cognitive radio (CR), spectrum sensing (SS), energy detection, CFAR, adaptive threshold, cyclostationarity (CS), OFDM.

\section{Introduction}

Many studies carried out in various countries show that a great portion of the licensed spectrum is severely underused [1], [2]. The unused resources referred to as spectrum holes can then be reused by secondary users called cognitive radios. In recent years, the Federal Communications Commission (FCC) has considered the use of cognitive radio (CR) technology as a new strategy [3]. Mitola [4], [5] introduced the dynamic spectrum access concept in cognitive radios and since then $\mathrm{CR}$ is considered as a way of increasing spectrum utilization of modern wireless technologies which received a lot of interest in recent years [6]-[9]. CR systems are equipped with the capability to adapt the transmission parameters according to the interactions with the surrounding radio environment to achieve predefined objectives. An interesting characteristic is that mobile and stationary terminals can adjust their parameters of 
transmission by the modification of the software embedded in the radio without any modifications on the hardware [10]. In the context of dynamic spectrum access [4], the secondary users (SUs) have no spectrum licenses but are allowed to use temporarily the licensed spectrum originally allocated to primary users (PUs) provided they avoid causing harmful interferences to the primary users. This opportunistic usage of the spectrum makes spectrum sensing (SS) an important and challenging topic in CR systems [11]. Indeed, SS is one of the most essential components and it has been of great interest recently [12], [13]. The crucial task of the SU is to obtain awareness about the spectrum usage and detection of the presence or absence of a PU's signals in a geographical area. Various SS methods have been proposed in the literature such as energy detectors (ED), waveform or matched filter based detectors, feature based detectors, and some emerging eigenvalue-based and wavelet-based detectors [10]. Each of these detectors has its advantages and disadvantages with varying detection capabilities, implementation complexity, sensing time, assumptions and requirements on the PU signal. For instance, the ED is simple to implement and it does not make any assumptions on the PU signal structure. In fact, the ED displays an effect known as "SNR wall" beyond which energy detection is theoretically infeasible [14]. Waveform or matched filter detection algorithms make explicit assumptions about the known pilot waveform or the exact transmitted signal which affect the confidentiality of the communication. Feature based detectors exploit only the structural or statistical properties of the PU signal. Since almost all man-made communication signals contain a redundancy added for example by coding, modulating, and burst formatting schemes used at the transmitter, they can be modeled as cyclostationary (CS) processes. Several works on CS based signal detection were proposed in [15]-[17]. In [18] a detection algorithm exploiting the property of boosted pilot subcarriers in OFDM signals is proposed where a two-threshold decision procedure based on the constant spacing property of spectral correlation at different cyclic frequencies was used. Another multi-cycle detector based on the Fourier representation of the cyclic autocorrelation function has been proposed in [19], the authors employ the test 
statistics as a ratio of two quadratic cyclic autocorrelation functions (CAF) exploiting the fact that the (CAF) is non-zero for some cyclic frequencies when an OFDM signal is present and zero otherwise because the noise is wide-sense stationary (WSS) with no correlation. In general, it is hard to detect OFDM signals using the general cyclostationary detection methods, which are robust to random noise at the cost of high computational complexity and long sensing times as the test statistics is computed for various values of the cyclic frequencies. To overcome such high computational complexities and long sensing times, we focus in this paper on spectrum sensing of OFDM signals by exploiting only the stationary part of the periodic autocorrelation function. This significantly simplifies the detector design and helps avoid the implementation of a large FFT operator as in [18] and [19]. Correlation based techniques are commonly used for hypothesis testing problem in various radar and communications applications. In wireless communications, it has been used in spread spectrum direct sequence code division multipleaccess DS-CDMA systems for the PN code synchronization [20] and [21], while in CRs, the design of detectors that utilize the built-in periodicity autocorrelation property of the CP-OFDM primary users have been derived in [22], [23], [24] and [25], and is also considered in this paper. Detection of OFDM signals in the region of low SNR is an important research problem and it has actually attracted significant attention [22]-[26]. In case of noise and signal powers are known, the optimal Neyman-Pearson (NP) spectrum sensing was derived and evaluated in [25]. The derived optimal NP detector needs a priori knowledge of the signal and noise power level to set the decision threshold, which unfortunately may not be available in practice. On the other hand, the ED does not require any a priori knowledge about the structure of detected signals or their parameters and it performs well if the noise power is perfectly known. However, even a small uncertainty in the noise power value causes severe performance degradation [27]. In addition, the spectrum sensing algorithm developed in [25] incorporates a non-declared synchronization mechanism, which result in more complexity and delay in the sensing task. These drawbacks of the above mentioned detectors motivated us to develop a fast and adaptive spectrum sensing 
algorithm based on the generalized likelihood ratio test (GLRT). An adaptive Constant False Alarm Rate (CFAR) thresholding approach is used based on the estimation of the noise power as a function of second order cyclic statistics of recorded signals as shown in the following sections. This capability of adaption to noise fluctuations by maintaining a constant false alarm rate CFAR (CFARness) is a much desired property in many applications and especially when detecting signals in unknown and fluctuating noise power environments [28]-[30].

In this paper, we investigate the performance of the proposed detector in terms of the probability of detection $\left(P_{d}\right)$ versus SNR for a given probability of false alarm $\left(P_{f a}\right)$. In addition, we study the performance of the proposed detector compared to the classical energy detector, the sliding window detector [24] and Axell's detector [25]. Numerical analysis shows that of the proposed detector is not only robust against varying noise or non-stationary interferences but also it achieves better detection performances than the existing detectors.

The rest of the paper is organized as follows. Section II introduces the data model and the spectrum sensing problem formulation in OFDM-based cognitive radio systems. Section III provides a short description of existing algorithms. Section IV describes in detail the proposed spectrum sensing algorithm. Section V describes the results of our performance analysis. Finally, Section VI draws the main conclusions.

\section{OFDM spectrum sensing background}

The basic concept of dynamic spectrum access is to unlock the licensed spectrum to secondary users under the condition that the interferences caused to primary users are limited [31]. The secondary user senses the spectrum to detect the presence or absence of the primary user in the licensed spectrum before data transmission. In addition, when the primary user reclaims its band during second user data transmission, the secondary user must interrupt transmitting data and vacate the spectrum as fast as possible. We assume that the primary and secondary users operate in the time domain and that the secondary users have data symbols to transmit. 


\subsection{OFDM-based system model}

The primary user transmitter is modeled as an orthogonal frequency division multiplexing (OFDM) based system with $N_{d}$ subcarriers, an OFDM signal consists of a sum of subcarriers that are modulated using phase shift keying (PSK) or qudrature amplitude modulation (QAM). Then one OFDM symbol of $N_{d}$ duration can easily obtained by introducing $N_{d}$ complex symbols $d_{i}, i=0, \ldots N_{d}-1$ to an $N_{d}$-point inverse discrete Fourier transform (IDFT) efficiently implemented through the fast Fourier transform (FFT) algorithm. Assuming that the sampling factor is 1 , the IDFT output can be written as

$$
s(n)=\sum_{i=0}^{N_{d}-1} d_{i} e^{j 2 \pi n * i / N_{d}} \quad 0 \leq n \leq N_{d}-1
$$

where, $n$ is a discrete time index, $N_{d}$ denotes the number of samples in an OFDM data block, $i / N_{d}$ is the $i$-th subcarrier frequency and $d_{i}, i=0, \ldots N_{d}-1$ is the PSK or QAM complex symbol modulating the $i$-th subcarrier. The IDFT data are then, converted to form a serial stream. Furthermore, to reduce the effect of ISI a guard time of length $N_{c}$ is introduced in every

OFDM symbol. The OFDM transmitter copies the last $N_{c}$ samples at the front of each OFDM symbol forming the overhead called cyclic prefix CP. This yields a new sequence of duration $N_{s}=N_{c}+N_{d}$ samples, which can be expressed as

$$
\boldsymbol{s}=\left[s(-N c) s(-N c+1) \cdots s(0) s(1) \cdots s\left(N_{d}-1\right)\right]^{T}
$$

where $T$ denotes transpose. Usually an OFDM frame contains several such blocks. The entire endless serial stream is transmitted over the wireless channel to the receiver.

Now denote by $\theta$ the time offset, i.e. the delay after which the secondary user receiver receives the first OFDM symbol. If we consider that the received signal contains $K$ OFDM symbols, we use an observation window of length $L$ samples $L=K\left(N_{d}+N_{c}\right)+N_{d}$ as illustrated in Fig. 1 . In an additive white Gaussian noise (AWGN) channel, the received signal at the secondary user can be written as

$$
y(k)=s(n)+n(k), \quad k=0,1, \cdots, K\left(N_{d}+N_{c}\right)+N_{d}-1 .
$$




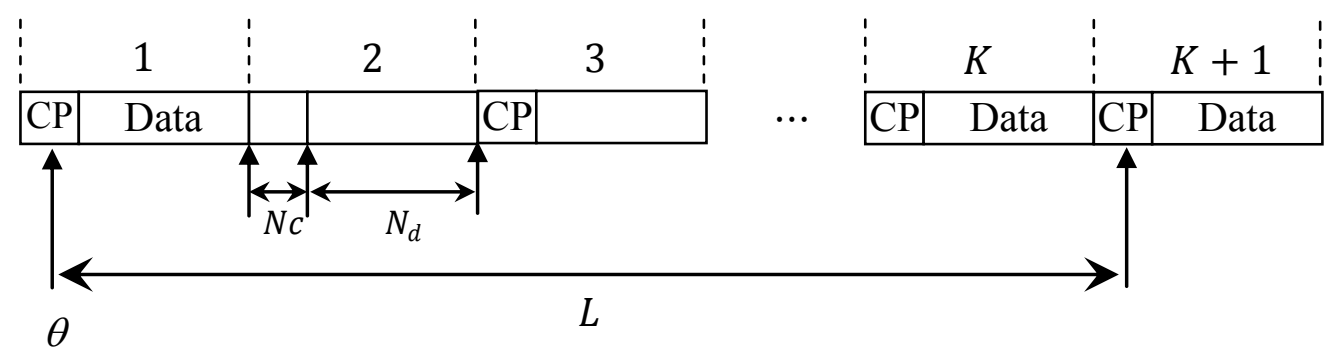

Fig. 1. OFDM signal structure with cyclic prefix CP.

\subsection{Spectrum sensing problem formulation}

Basically, the problem of spectrum sensing is to decide whether a transmitted signal is present or not, based on the received signal. That is, it can be formulated as a binary hypothesis testing problem, where we need to discriminate between the two hypotheses:

$$
\begin{cases}H_{1}: y(k)=s(n)+n(k), & k=0,1, \cdots, K\left(N_{d}+N_{c}\right)+N_{d}-1 \\ H_{0}: y(k)=n(k), & k=0,1, \cdots, K\left(N_{d}+N_{c}\right)+N_{d}-1 .\end{cases}
$$

where $H_{1}$ and $H_{0}$ correspond to presence and absence of an OFDM signal respectively. The number of samples collected during a sensing interval is $L=K\left(N_{d}+N_{c}\right)+N_{d}$. In the proposed model, we have assumed an AWGN channel, i.e. the noise is complex white zero-mean Gaussian with variance $\sigma_{n}^{2}$. Under the assumption of a sufficiently large IFFT size and according to the central limit theory, the signal is also assumed complex white zero-mean Gaussian with variance $\sigma_{s}^{2}$. In practice, sensing errors are inevitable due to additive noise, limited time observations, and the inherent randomness of the observed data. False alarms occur if an idle channel is detected as busy. On the other hand, missed detections occur when a busy channel is detected as idle. Consequently, a false alarm may lead to a potentially missed transmission opportunity for the secondary user, which results in a lower spectrum utilization. In addition, a missed detection could potentially lead to a collision with the primary user, leading to wasted transmissions for both PU and SU. Based on the above definitions of false alarms and missed detections, the performance of any spectrum sensing detector are characterized by two parameters, the probability of detection $P_{d}=1-P_{m d}=P\left(H_{1} / H_{1}\right)$, where $P_{m d}$ is the 
probability of missed detection and the probability of false alarm $P_{f a}=P\left(H_{1} / H_{0}\right)$. In addition, due to lack of information about the primary user activity, it is difficult to have a knowledge of the a priori probabilities $P\left(H_{0}\right)$ and $P\left(H_{1}\right)=1-P\left(H_{0}\right)$. Therefore, the design of the optimal spectrum sensing detector is based on the Neyman-Pearson (NP) hypothesis test, which maximizes $P_{d}$ for a given $P_{f a}[29]$.

\section{Survey of the spectrum sensing algorithms}

The algorithms discussed in this section range from energy detectors to feature detectors. A common point to all the feature detectors discussed here is the exploitation of some known structure of the transmitted signal.

\subsection{Energy detector}

An energy detector (ED) simply measures the received energy during a finite time interval called sensing time, and compares the estimated energy to a predetermined threshold. The decision rule of the ED is given by [32]

$$
T_{E D}=\|y\|^{2}=\sum_{i=1}^{N}\left|y_{i}\right|^{2} \underset{H 0}{\stackrel{H 1}{>}} \lambda_{E D}
$$

where $\left|y_{i}\right|$ denotes the module of the measured signal $y_{i}, i=1,2, \cdots, N$, which is given by

$$
\left|y_{i}\right|^{2}=\left(y_{i}^{I}\right)^{2}+\left(y_{i}^{Q}\right)^{2} \quad i=1,2, \ldots, N \text {. }
$$

$y_{i}^{I}$ and $y_{i}^{Q}$ are the in-phase and quadrature phase components respectively, and $\lambda_{E D}$ is the threshold chosen to satisfy a desired $P_{f a}$. The ED is a universal detector in the sense that the decision on the presence of a PU is obtained by comparing the received signal energy with a threshold, but it does not differentiate the PU from other unknown signal sources. Besides its low computational complexity, the design of a good energy detector is a challenging task. In low signal-to-noise ratio (SNR) regimes and in the presence of noise uncertainty, it is not possible to detect the primary signal at SNR values lower than a given value called the "SNR wall" even if the sensing time goes to infinity [27]. In addition, the detection threshold depends on the noise power, which may change over time and this may trigger false alarms frequently and/or a 
low $P_{d}$. All these issues motivated the search for a new SS technique that can exploit additional signal features along with a capacity of adapting the threshold to a varying noise environment which will be presented in Section IV.

\subsection{CP based sliding window detector}

The original sliding window (SW) detector used for sensing Digital Video Broadcasting Terrestrial (DVB-T) signals was proposed in [24]. It exploits the correlation property of OFDM signals. For only one ODFM symbol, a sliding window of size $N_{c}$ is used to compute the absolute value of the sum of the estimated autocorrelation over $N_{c}$ consecutive samples and then to take the maximum of the $N_{c}+N_{d}$ obtained statistics corresponding to the possible values of $\theta$ by moving the window by one sample each time to obtain the decision rule given by

$$
T_{S W}=\max _{\theta \in\left\{0, \ldots, N_{c}+N_{d}-1\right\}}\left|\sum_{j=\theta}^{\theta+N_{c}-1} \hat{r}_{j}\right| \begin{aligned}
& \mathrm{H}_{1} \\
& \underset{\mathrm{H}_{0}}{\gtrless}
\end{aligned} \lambda_{S W},
$$

where $\hat{r}_{j}=y_{j} y_{j+N_{d}},|\cdot|$ is the modulo operator and $\theta$ is the time when the first sample is observed or equivalently the synchronization mismatch between the transmitter and the receiver. A generalization of this detector for the case of $K$ OFDM symbols using the whole signal and not one symbol only is obtained as [33]:

$$
T_{S W}=\max _{\theta \in\left\{0, \ldots, N_{c}+N_{d}-1\right\}}\left|\sum_{j=\theta}^{\theta+N_{c}-1} \hat{R}_{j}\right| \begin{gathered}
\mathrm{H}_{1} \\
\mathrm{H}_{0}
\end{gathered} \lambda_{S W},
$$

where $\hat{R}_{j}=\frac{1}{K} \sum_{k=0}^{K-1} \hat{r}_{j+k}\left(N_{d}+N_{c}\right)$. However, the test statistic and the threshold of the detectors in (7) or (8) are affected by a scaling of the problem such as multiplying the received data by a constant. Hence the main drawback of these detectors is the knowledge of the noise variance $\sigma_{n}^{2}$ to set the decision threshold $\lambda_{S W}$, which may not be available.

\subsection{Axell's detector}

In [25], a (GLRT) based spectrum sensing for CR systems is proposed based on the second order statistics of the observed signals, when the signal power $\sigma_{s}^{2}$, noise power $\sigma_{n}^{2}$, and 
synchronization mismatch $\theta$ are unknown. The proposed detector employs the following decision strategy:

$$
T_{A X}=\max _{\theta \in\left\{0, \ldots, N_{c}+N_{d}-1\right\}} \frac{\sum_{j=0}^{N_{d}+N_{c}-1}\left|\hat{R}_{j}\right|^{2}}{\sum_{j \in C P}\left|\hat{R}_{j}-\frac{1}{N_{c}} \sum_{k \in C P} \overline{\widehat{R}}_{k}\right|^{2}+\sum_{j \notin C P}\left|\hat{R}_{j}\right|^{2}} \underset{\mathrm{H}_{0}}{\gtrless} \lambda_{G L R T}
$$

$\overline{\hat{R}}_{k}$ represents the real part of the correlation $\hat{R}_{k}$ over $K$ OFDM symbols, $C P$ are the indices of the $N_{c}$ consecutive correlated OFDM samples given $\theta$, while $\hat{R}_{k}$ is obtained by

$$
\hat{R}_{j}=\frac{1}{K} \sum_{k=0}^{K-1} y_{j+k N_{s}} y_{j+k N_{s}+N_{d}}^{*} ; j=0,1, \ldots, N_{s}-1
$$

$K$ is the number of OFDM symbols in the observation window. We observe, however, that unlike the energy detector the test statistic of Axell's detector (Eqn. 9) has a CFAR property since it is not affected by the term containing the received data multiplied by a constant, and hence in this case the decision threshold can be set for a desired false alarm probability without knowledge of the noise power.

\section{A CS-based Spectrum Sensing Algorithm}

In this section, we derive a spectrum sensing algorithm by exploiting the cyclostationarity property of the OFDM signals by means of a GLRT approach. The GLRT is a likelihood ratio test where the unknown parameters are replaced by their maximum-likelihood (ML) estimates [29]. The test statistic is derived under the assumptions that:

(i) The noise variance $\sigma_{n}^{2}$ and signal variance $\sigma_{s}^{2}$ are unknown.

(ii) The receiver is not synchronized to the transmitter $(\theta \neq 0)$, that is the receiver does not know the starting point of each OFDM symbol as shown in Fig. 1.

(iii) The parameters $N_{d}$ and $N_{c}$ are known. This is a reasonable assumption about the PU waveforms since this is specified by the standards and can therefore be assumed to be known to the detector. 
(iv) At the receiver, we have $K$ observed consecutive OFDM symbols out of the stream of OFDM modulated data, so that the received signal contains $L=K\left(N_{d}+N_{c}\right)+N_{d}$ samples.

We define the sample value products $r_{n}$ of two samples of the received signal $y_{n}$ and $y_{n+N_{d}}$ with distance $N_{d}$ over the observation window of length $L$ as

$$
\hat{r}_{n}=y_{n} y_{n+N_{d}}, \quad n=0, \ldots, K\left(N_{d}+N_{c}\right)-1
$$

Exploiting the periodicity of the autocorrelation function in the case of OFDM signals that is due to the presence of the cyclic prefix $(\mathrm{CP})$ in the data. This period is equal to one OFDM symbol of length $N_{s}=N_{c}+N_{d}$. Assuming that the OFDM symbols are independent and averaging the second order statistics of (11) over $K$ multiple OFDM symbols, we obtain the measure of the correlation between two samples with distance $N_{d}$ during one period defined by

$$
\hat{R}_{i}=\frac{1}{K} \sum_{k=0}^{K-1} \hat{r}_{i+k\left(N_{d}+N_{c}\right)}, \quad i=0, \ldots, N_{d}+N_{c}-1
$$

In the binary hypothesis detection problem considered, all the values $\hat{R}_{i}$ under $H_{0}$ are identically distributed since the received signal consists of noise only without $\mathrm{CP}$, while under $H_{1}$ and due to the repeated data caused by the insertion of the CP with length $N_{c}$ there will be $N_{c}$ values of $\hat{R}_{i}$ with high correlation starting at the value $\theta$ and the other $N_{d}$ values have negligible correlation. In Fig. 2, we illustrate this property in the presence of an OFDM signal with $N_{d}=32$ (IFFT block size), cyclic prefix $N_{c}=N_{d} / 4=8$, number of OFDM symbols is $K=500$ and the first OFDM symbol is assumed to be received at some instant, say $\theta=19$.

The eight samples corresponding to the CP region of any OFDM symbol exhibit stronger correlation values than the others. Hence, when an OFDM signal is present the autocorrelation function has peak values at the time instants corresponding to the CP region. On the other hand, noise or signals without this property of cyclic-correlation induced by $\mathrm{CP}$ are uncorrelated and the correlation values will be small. 


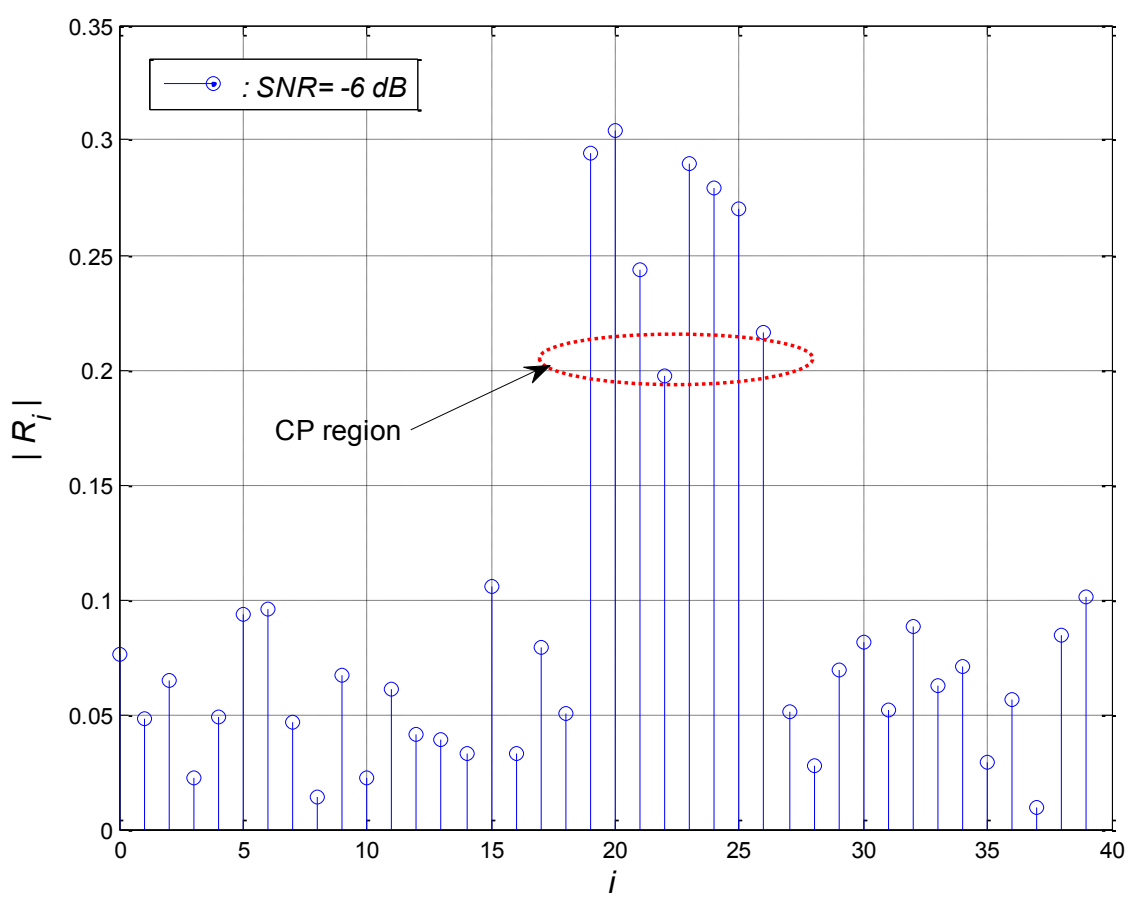

Fig. 2. Example of the autocorrelation function of the received OFDM signal with CP.

Based on this observation, we propose a detector that is able to distinguish an OFDM signal from noise, taking into consideration the practical difficulty that the detector does not know the starting point of an OFDM symbol $\theta$, the signal power $\sigma_{s}^{2}$ and the noise power $\sigma_{n}^{2}$.

We define the correlation vector $\widehat{\boldsymbol{R}}=\left[\widehat{R}_{0} \hat{R}_{1} \cdots \hat{R}_{N_{d}+N_{c}-1}\right]^{T}$ and calculate the natural logarithm of the likelihood ratio (LLR):

$$
\Lambda_{\mathrm{g}}(\widehat{\mathbf{R}})=\ln \left(\frac{\max _{\theta, \sigma_{n}^{2}, \sigma_{s}^{2}} f_{\widehat{\boldsymbol{R}} / H_{1}}\left(\widehat{\boldsymbol{R}} / H_{1}, \theta, \sigma_{n}^{2}, \sigma_{s}^{2}\right)}{\max _{\sigma_{n}^{2}} f_{\widehat{\boldsymbol{R}} / H_{0}}\left(\widehat{\boldsymbol{R}} / H_{0}, \sigma_{n}^{2}\right)}\right)=\max _{\theta} \ln \left(\frac{f_{\widehat{\boldsymbol{R}} / H_{1}}\left(\widehat{\boldsymbol{R}} / H_{1}, \theta, \widehat{\sigma_{n}^{2}}, \widehat{\sigma_{s}^{2}}\right)}{f_{\widehat{\boldsymbol{R}} / H_{0}}\left(\widehat{\boldsymbol{R}} / H_{0}, \widehat{\sigma_{n}^{2}}\right)}\right) \underset{\mathrm{H}_{0}}{\stackrel{\mathrm{H}_{1}}{\gtrless} \lambda}
$$

where $\ln (\cdot)$ denotes the natural logarithm, $\hat{\alpha}$ is the ML estimate of the parameter $\alpha$ and $\lambda$ is the decision threshold. For simplicity, we assume that $\hat{R}_{i}$ are independent, so that the probability density functions (pdf's) of (13) can be written as

$$
f_{\widehat{\boldsymbol{R}} / H_{i}}\left(\widehat{\boldsymbol{R}} / H_{i}, \theta, \sigma_{n}^{2}, \sigma_{s}^{2}\right)=\prod_{k=0}^{N_{d}+N_{c}-1} f_{\widehat{R}_{k} / H_{i}}\left(\hat{R}_{k} / H_{i}, \theta, \sigma_{n}^{2}, \sigma_{s}^{2}\right), \quad i=0,1 .
$$

Since $\hat{R}_{k}, k=0,1, \ldots, N_{d}+N_{c}-1$, are complex random variables, they can be defined as the sum of two real random variables (RV's) $\overline{\hat{R}}_{k}$ and $\widetilde{\hat{R}}_{k}$, i.e., $\hat{R}_{k}=\overline{\hat{R}}_{k}+j \widetilde{\hat{R}}_{k}$ as in [25], then we have 


$$
\overline{\hat{R}}_{k}=\frac{1}{K} \sum_{l=0}^{K-1} \overline{\hat{r}}_{k+l\left(N_{d}+N_{c}\right)}, \quad k=0, \ldots, N_{d}+N_{c}-1
$$

and

$$
\tilde{\hat{R}}_{k}=\frac{1}{K} \sum_{l=0}^{K-1} \tilde{\hat{r}}_{k+l\left(N_{d}+N_{c}\right)}, \quad k=0, \ldots, N_{d}+N_{c}-1
$$

The conditional pdf's given in (14) can be rewritten using (15) and (16) as follows:

$$
f_{\widehat{\boldsymbol{R}} / H_{i}}\left(\widehat{\boldsymbol{R}} / H_{i}, \theta, \sigma_{n}^{2}, \sigma_{s}^{2}\right)=\prod_{\mathrm{k}=0}^{N_{d}+N_{c}-1} f_{\bar{R}_{k}, \tilde{R}_{k} / H_{i}}\left(\overline{\hat{R}}_{k}, \tilde{\hat{R}}_{k} / H_{i}, \theta, \sigma_{n}^{2}, \sigma_{s}^{2}\right), \quad i=0,1
$$

Hence, we need to determine the joint pdf's of $\widehat{\hat{R}}_{k}$ and $\widetilde{\hat{R}}_{k}$ under the hypotheses $H_{0}$ and $H_{1}$.

\subsection{Distribution of $\overline{\hat{R}}_{i}$ and $\tilde{\hat{R}}_{i}$ under hypotheses $H_{0}$ and $H_{1}$}

In CR systems, the sensing time defined in many wireless standards is usually at the level of hundreds of milliseconds, which corresponds to an observation window with hundreds to thousands of OFDM symbols. Under these conditions $\overline{\hat{R}}_{k}$ and $\widetilde{\hat{R}}_{k}$ can be assumed to be a Gaussian distributed random variables based on the central limit theory. Moreover, we use the Gaussian approximation to model the complex random variables $\hat{R}_{i}, i=0,1, \ldots, N_{d}+N_{c}-1$, (see [22], [23], and [25]) to derive a closed form expression of the log-likelihood ratio test. In addition, the detection of OFDM signals is more crucial at low-SNR $\left(\sigma_{n}^{2}>>\sigma_{s}^{2}\right)$ and hence we use a low-SNR approximation to simplify the computation of the ML estimates. In low-SNR

regime, $\overline{\hat{R}}_{k}$ and $\widetilde{\hat{R}}_{k}$ are uncorrelated Gaussian RV's [25]. Hence, the pdf's of $\overline{\hat{R}}_{k}$ and $\widetilde{\hat{R}}_{k}$ under hypothesis $H_{0}$ are given by

and

$$
f_{\bar{R}_{k} / H_{0}}\left(\overline{\hat{R}}_{k} / H_{0}\right)=\frac{1}{\sqrt{2 \pi} \sigma_{0}} \exp \left(-\frac{\left(\widehat{\hat{R}}_{k}\right)^{2}}{2 \sigma_{0}^{2}}\right), \quad k=0,1, \cdots, N_{d}+N_{c}-1
$$

$$
f_{\tilde{R}_{k} / H_{0}}\left(\tilde{\hat{R}}_{k} / H_{0}\right)=\frac{1}{\sqrt{2 \pi} \sigma_{0}} \exp \left(-\frac{\left(\tilde{\hat{R}}_{k}\right)^{2}}{2 \sigma_{0}^{2}}\right), \quad k=0,1, \cdots, N_{d}+N_{c}-1
$$

where $\sigma_{0}^{2}=\sigma_{n}^{4} / 2 K, \sigma_{n}^{2}$ is the noise power and $K$ is the number of OFDM symbols. Under hypothesis $H_{1}$, we have two possible cases. In the first one, an OFDM signal is present and the 
signal samples are equal, i.e. $k$ falls in the CP region as shown in Fig. 2. In this case the pdf's of $\widehat{\hat{R}}_{k}$ are given by

$$
f_{\bar{R}_{k} / H_{1}}\left(\overline{\hat{R}}_{k} / H_{1}\right)=\frac{1}{\sqrt{2 \pi} \sigma_{0}} \exp \left(-\frac{\left(\overline{\hat{R}}_{k}-\sigma_{s}^{2}\right)^{2}}{2 \sigma_{0}^{2}}\right), \quad k \in \text { CP region }
$$

where $\sigma_{s}^{2}$ is the power of the received signal. In the second case, an OFDM signal is present but $k$ is not in the CP region and the resulting pdf's of $\overline{\hat{R}}_{k}$ are given by

$$
f_{\overline{\hat{R}}_{k} / H_{1}}\left(\widehat{\widehat{R}}_{k} / H_{1}\right)=\frac{1}{\sqrt{2 \pi} \sigma_{0}} \exp \left(-\frac{\left(\widehat{\widehat{R}}_{k}\right)^{2}}{2 \sigma_{0}^{2}}\right), \quad k \notin \text { CP region }
$$

We note that the pdf's of $\tilde{\hat{R}}_{k}$ under hypothesis $H_{1}$ are independent of $k$ and are given by

$$
f_{\tilde{R}_{k} / H_{1}}\left(\tilde{\hat{R}}_{k} / H_{1}\right)=\frac{1}{\sqrt{2 \pi} \sigma_{0}} \exp \left(-\frac{\left(\tilde{\hat{R}}_{k}\right)^{2}}{2 \sigma_{0}^{2}}\right), \quad k=0,1, \cdots, N_{d}+N_{c}-1
$$

From (19) and (22) we observe that the random variables $\widetilde{\hat{R}}_{k}$ have the same probability density function for $k=0,1, \cdots, N_{d}+N_{c}-1$ and do not depend on which hypothesis is true. Only $\widehat{\widehat{\mathrm{R}}}_{\mathrm{k}}$ for $\mathrm{k}=0,1, \cdots, N_{d}+N_{c}-1$ depend on the hypothesis $H_{1}$ and $H_{0}$. That is the imaginary part of the correlation $\tilde{\hat{R}}_{k}$ is not a discriminating function which leads to a "nice" simplification of the ratio test as will be shown in the next section. An intuitive interpretation of the obtained result is as follows. Since the imaginary part of the correlation $\widetilde{\mathrm{R}}_{\mathrm{k}}$ is a measure of the cross-correlation between the in-phase and quadrature phase components of the received signal which are independent and identically distributed, using the central limit theorem and in particular in the low SNR region considered in the paper, this cross-correlation will have the same distribution (mean zero and same variance) under both hypotheses $H_{0}$ and $H_{1}$. This is a direct result of the independence of the quadrature components of the signal and thus the crosscorrelation is not a discriminating function.

\subsection{CS-based sensing detector}

Since $\overline{\hat{R}}_{k}$ and $\widetilde{\hat{R}}_{k}$ are uncorrelated Gaussian RV's [25], the joint pdf of $\widehat{\boldsymbol{R}}$ in (17) becomes 


$$
\begin{aligned}
& f_{\widehat{\boldsymbol{R}} / H_{i}}\left(\widehat{\boldsymbol{R}} / H_{i}, \theta, \sigma_{n}^{2}, \sigma_{s}^{2}\right)= \\
& \prod_{k=0}^{N_{d}+N_{c}-1} f_{\bar{R}_{k} / H_{i}}\left(\overline{\hat{R}}_{k} / H_{i}, \theta, \sigma_{n}^{2}, \sigma_{s}^{2}\right) f_{\tilde{R}_{k} / H_{i}}\left(\tilde{\hat{R}}_{k} / H_{i}, \theta, \sigma_{n}^{2}, \sigma_{s}^{2}\right), \quad i=0,1 .
\end{aligned}
$$

Substituting (23) in (13), the LLRT we get:

$$
\begin{array}{r}
\Lambda_{\mathrm{g}}(\widehat{\mathbf{R}})=\max _{\theta} \ln \left(\frac{\prod_{k=0}^{N_{d}+N_{c}-1} f_{\bar{R}_{k} / H_{1}}\left(\overline{\hat{R}}_{k} / H_{1}, \theta, \widehat{\sigma_{n}^{2}}, \widehat{\sigma_{s}^{2}}\right) \prod_{k=0}^{N_{d}+N_{c}-1} f_{\tilde{R}_{k} / H_{1}}\left(\tilde{\hat{R}}_{k} / H_{1}, \theta, \widehat{\sigma_{n}^{2}}, \widehat{\sigma_{s}^{2}}\right)}{\prod_{k=0}^{N_{d}+N_{c}-1} f_{\overline{\widehat{R}}_{k} / H_{0}}\left(\overline{\hat{R}}_{k} / H_{0}, \widehat{\sigma_{n}^{2}}\right) \prod_{k=0}^{N_{d}+N_{c}-1} f_{\tilde{R}_{k} / H_{0}}\left(\tilde{R}_{k} / H_{0}, \widehat{\sigma_{n}^{2}}\right)}\right) \\
=\max _{\theta} \ln \left(\frac{\prod_{k=0}^{N_{d}+N_{c}-1} f_{\overline{\hat{R}}_{k} / H_{1}}\left(\overline{\hat{R}}_{k} / H_{1}, \theta, \widehat{\sigma_{n}^{2}}, \widehat{\sigma_{s}^{2}}\right)}{\prod_{k=0}^{N_{d}+N_{c}-1} f_{\bar{R}_{k} / H_{0}}\left(\overline{\hat{R}}_{k} / H_{0}, \widehat{\sigma_{n}^{2}}\right)}\right) \underset{\mathrm{H}_{0}}{\gtrless} \lambda
\end{array}
$$

From (24), we observe that the test static depends only of the real part $\overline{\hat{R}}_{k}$ of the complex RV's $\hat{R}_{k}$, whereas the imaginary part doesn't contribute or add any information in obtaining the sufficient statistic, unlike the decision rule obtained in [25], which was dependent on both the real and imaginary parts. This is because the imaginary parts of $\hat{R}_{k}$ have the same distributions under both hypotheses. Fig. 3 illustrates nicely the fact that only the real parts $\overline{\hat{R}}_{k}$ have a distribution that depends on $k$ and the imaginary parts distribution of $\overline{\hat{R}}_{k}$ are independent on $\mathrm{k}$.

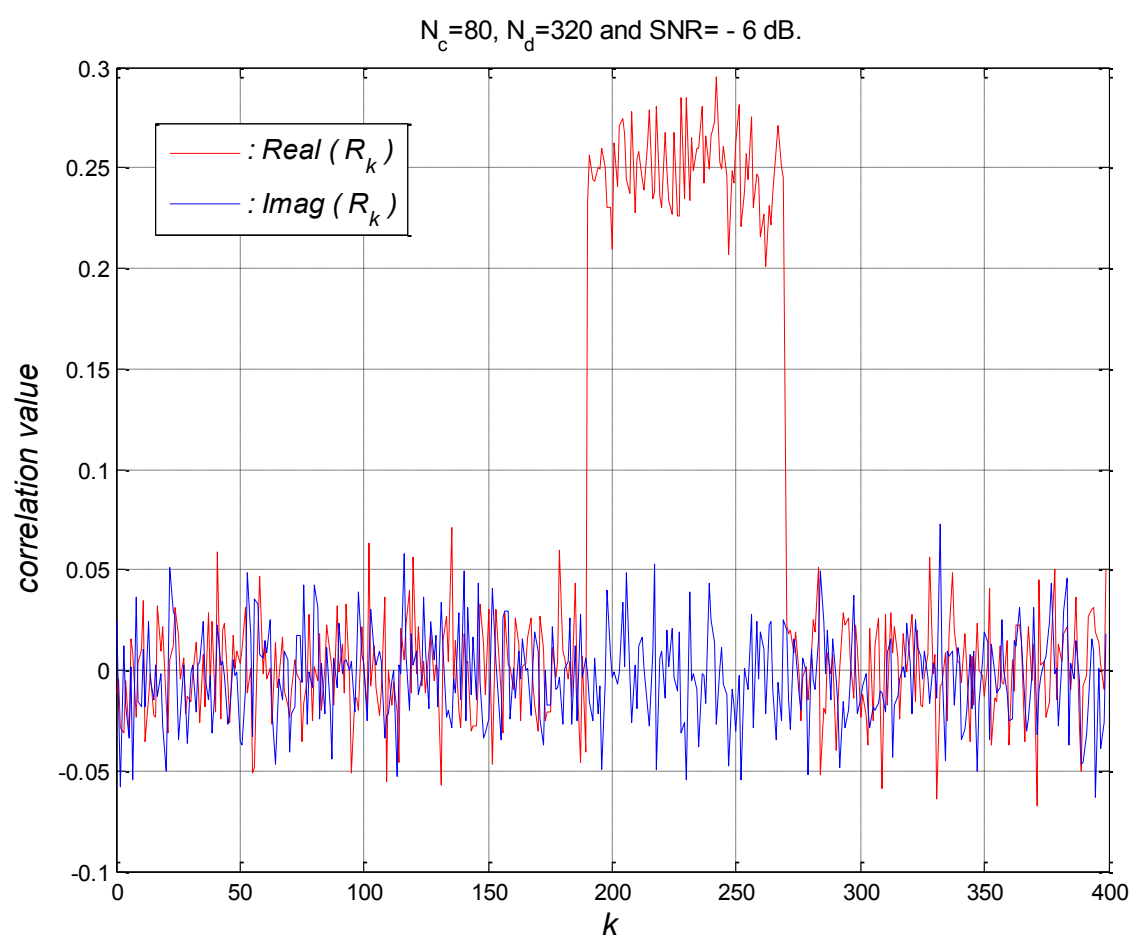

Fig. 3. Real and imaginary parts of the correlation function of an OFDM signal. 
Substituting (18), (20), and (21) in (23) we obtain the pdf's of the real part $\bar{R}_{k}$ under both hypotheses $H_{0}$ and $H_{1}$ as

$$
\prod_{k=0}^{N_{d}+N_{c}-1} f_{\bar{R}_{k} / H_{0}}\left(\overline{\hat{R}}_{k} / H_{0}, \sigma_{n}^{2}\right)=\frac{1}{\left(\sqrt{2 \pi} \sigma_{0}\right)^{N_{d}+N_{c}}} \exp \left(-\frac{1}{2 \sigma_{0}^{2}} \sum_{k=0}^{N_{d}+N_{c}-1}\left(\overline{\hat{R}}_{k}\right)^{2}\right)
$$

and

$$
\begin{aligned}
& \prod_{k=0}^{N_{d}+N_{c}-1} f_{\bar{R}_{k} / H_{1}}\left(\overline{\hat{R}}_{k} / H_{1}, \theta, \sigma_{n}^{2}, \sigma_{s}^{2}\right)= \\
& \frac{1}{\left(\sqrt{2 \pi} \sigma_{0}\right)^{N_{d}+N_{c}}} \exp \left(-\frac{\sum_{k \in C P}\left(\overline{\hat{R}}_{k}-\sigma_{\mathrm{s}}^{2}\right)^{2}+\sum_{l \notin C P}\left(\overline{\hat{R}}_{l}\right)^{2}}{2 \sigma_{0}^{2}}\right)
\end{aligned}
$$

It is easy to show that the ML estimate of the signal variance $\sigma_{s}^{2}$ is

$$
\widehat{\sigma_{s}^{2}}=\frac{1}{N_{c}} \sum_{k \in C P} \widehat{\hat{R}}_{k}
$$

For the estimation of $\sigma_{0}^{2}$, we consider only the $N_{d}$ random variables $\overline{\hat{R}}_{k}$ that do not lie in the CP region, and thus the ML estimate of $\sigma_{0}^{2}$ under the two test hypothesis signal absent $\left(\mathrm{H}_{0}\right)$ and signal present $\left(\mathrm{H}_{1}\right)$ respectively are the same given by

$$
\widehat{\sigma_{0}^{2}} / H_{0}=\widehat{\sigma_{0}^{2}} / H_{1}=\frac{1}{N_{d}} \sum_{\substack{k=0 \\ k \notin C P}}^{N_{d}+N_{c}-1}\left(\bar{R}_{k}\right)^{2}
$$

We observe also that the choice of taking $N_{d}$ samples (i.e. data outside the CP) to estimate $\sigma_{0}^{2}$ is really judicious because with having the same estimate of $\sigma_{0}^{2}$ for the $N_{d}$ samples resulted in a much simple decision rule as given in (28). Substituting for the estimated values of $\sigma_{0}^{2}$ and $\sigma_{s}^{2}$ in (25) and (26) and after some mathematical manipulations we get the following decision rule:

$$
\Lambda_{\mathrm{g}}(\overline{\widehat{\boldsymbol{R}}})=\max _{\theta \in\left\{0, \ldots, N_{c}+N_{d}-1\right\}}\left(\sum_{k \in C P} \overline{\hat{R}}_{k}\right)^{2} \underset{\mathrm{H}_{0}}{\stackrel{\mathrm{H}_{1}}{\gtrless}} \eta \widehat{\sigma_{0}^{2}}
$$

The interpretation of (29) says that a synchronization process is implicitly incorporated in the detection algorithm and the maximum correlation is obtained when perfect synchronization 
occurs. To override the need of (implicit) synchronization, we can order the $\overline{\hat{R}}_{k}$ 's in an ascending order and take only the $N_{c}$ largest samples as follows:

$$
\Lambda_{\mathrm{g}}(\overline{\widehat{\boldsymbol{R}}})=\left(\sum_{j=N_{d}}^{N_{d}+N_{c}-1} \overline{\hat{R}}_{o s, j}\right)^{2} \underset{\mathrm{H}_{0}}{\stackrel{\mathrm{H}_{1}}{\gtrless}} \eta \widehat{\sigma_{0}^{2}}
$$

The obtained test statistic $\Lambda_{\mathrm{g}}(\overline{\boldsymbol{R}})$ and the threshold $\eta \widehat{\sigma_{0}^{2}}$ are unaffected by a scaling of the problem such as multiplying the received data by a constant, then the detector has a CFAR property where the threshold can be set for a desired $P_{f a}$ without knowledge of $\sigma_{n}^{2}$. This is easily shown by normalizing the received data $y_{n}$ in (4) by $\sigma_{n}$ to obtain $x_{n}=y_{n} / \sigma_{n}$. Exploiting the fact that $y_{n}$ are Gaussian with mean zero and variance $\sigma_{n}^{2}$ we obtain the probability density function of $x_{n}$ as $f_{X_{n} / H_{0}}\left(x_{n} / H_{0}\right)=1 / \sqrt{2 \pi} \exp \left(-x_{n}^{2} / 2\right)$ which are independent of the noise power $\sigma_{n}^{2}$ under $H_{0}$ in the case of receiving just noise. Replacing $y_{n}$ by $x_{n}$ in the decision rule given by (29) the parameter $\sigma_{n}^{4}$ simplified from both sides of (29) and the probability of false alarm $\left(P_{f a}\right)$ becomes equal to Prob $\left\{\Lambda_{g}(\overline{\hat{R}})>\eta \widehat{\sigma_{0}^{2}} / H_{0}\right\}$ which is independent of the noise power and thus the detector has the desired CFAR property since the threshold can be set from the design $P_{f a}$ without knowledge of $\sigma_{n}^{2}$ as just mentioned above. We observe also that the test obtained is more computationally efficient than the one in (29), since it does not require any information about the timing synchronization parameter $\theta$. This results in a reduced sensing time, obtained after removing the serial search process implemented as a sliding window. This represents an interesting property especially for real time implementation of the algorithm. To validate the proposed ranking procedure, we compare the decision statistic of (30) with that of (29), which is implemented as a sliding window.

In the simulations, we chose the number of subcarriers $N_{d}=32$, the CP length $N_{c}=N_{d} / 4=8$, the number of OFDM symbols $K=500$, and the first OFDM symbol received at an instant $\theta=$ 19. We observe from Fig. 4 that the decision statistics are very close to each other for $S N R S$ higher than $-15 d B$. 


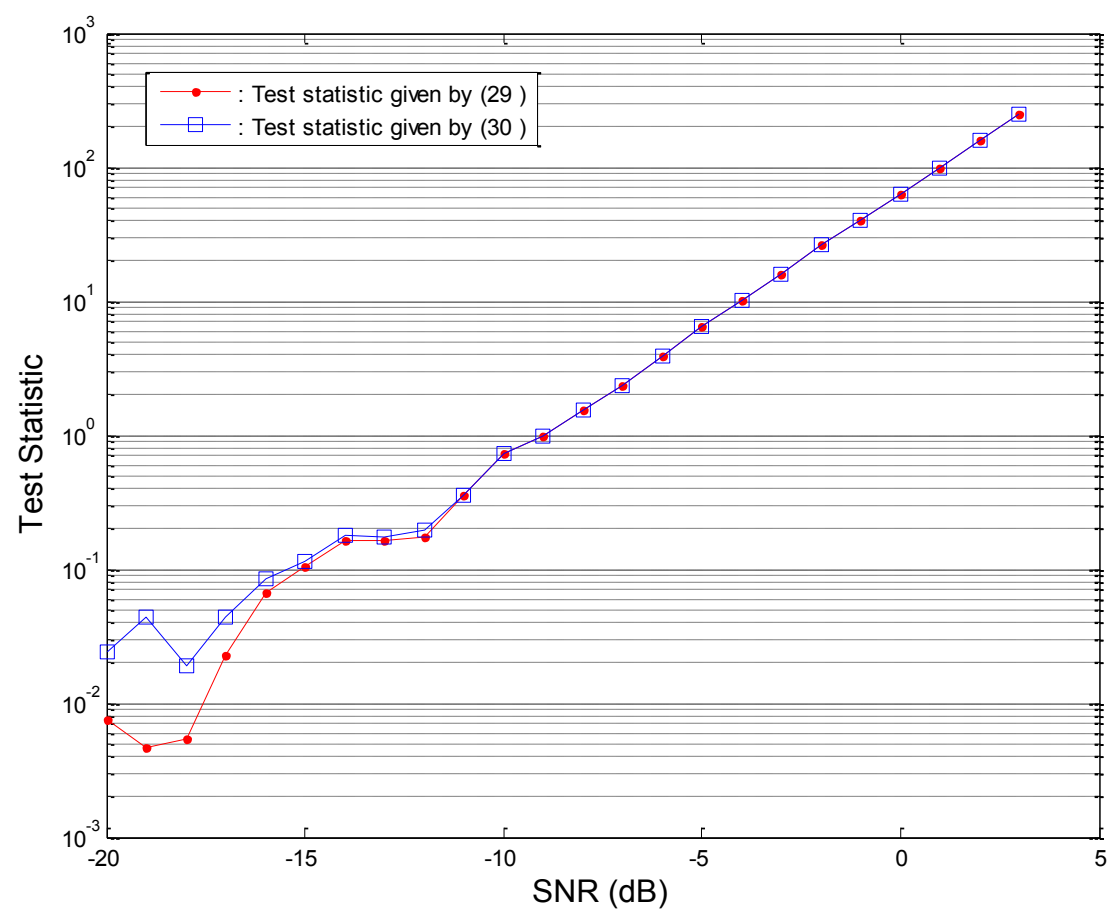

Fig. 4. Comparison of the test statistics in (29) and (30) as a function of the SNR.

The proposed algorithm for the detection of OFDM signals can be summarized as follows:

(i) Using the observed samples during a window with length of $K$ OFDM symbols, we compute $N_{c}+N_{d}$ values of the real part of the autocorrelation function at time lag $N_{d}$. We call these values correlation bins, which correspond to one period of the autocorrelation function defined in (15), this terminology is widely used in radar nomenclature.

(ii) The output of the correlator representing the correlation bins are sent serially into a shift register of length $N_{c}+N_{d}$.

(iii) The $N_{c}+N_{d}$ cells are ranked in an ascending order according to their magnitude and a test statistic is formed from the upper bound values of $\bar{R}_{k}$, while the other lower values are used to form an estimate of the background noise level $\widehat{\sigma_{0}^{2}}$. This estimate is then multiplied by a constant $\eta$ called threshold multiplier, which is selected so that to achieve a desired false alarm probability $P_{f a}$.

(iv) Finally, we compare the computed sufficient statistic $\Lambda_{\mathrm{g}}(\overline{\boldsymbol{R}})$ given by (30) with the obtained threshold $\eta \widehat{\sigma_{0}^{2}}$ in order to make a decision about the presence or absence of an OFDM signal, as shown in Fig. 5. 


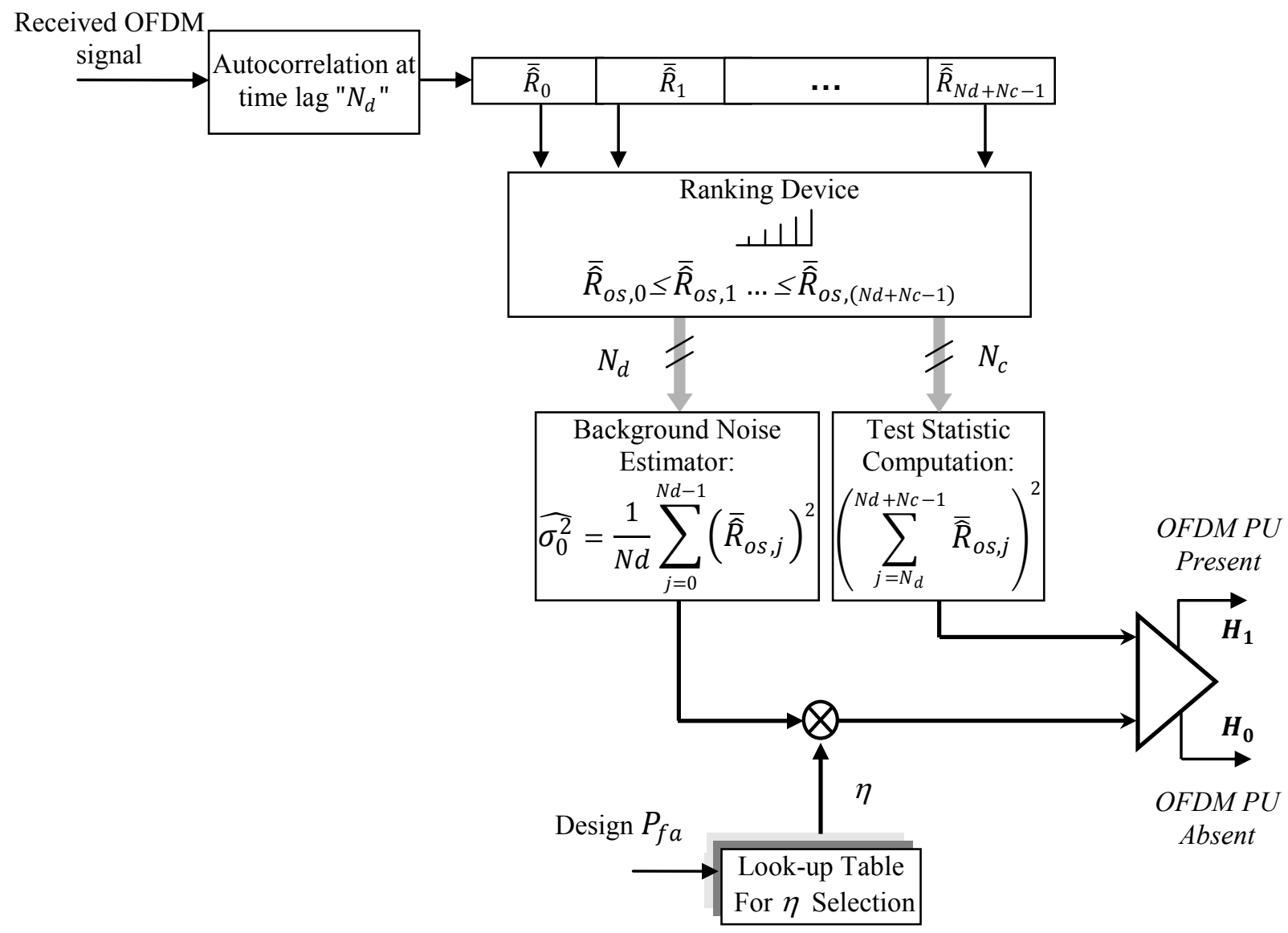

Fig. 5. Proposed CFAR spectrum-sensing algorithm for OFDM signal detection.

The proposed test in [25] is more computationally heavy than the proposed one. Since in [25], we first need to compute the empirical averages $R_{i}$ and then compute the LRT for each synchronization mismatch value $\theta, 0 \leq \theta<N_{c}+N_{d}$, and after that we take the maximum as the test statistic. Recall that in the proposed spectrum sensing detector the prior information about the synchronization parameter $\theta$ is not necessary, since the decision is reliably made in one scan instead of $\left(N_{c}+N_{d}\right)$ scans. This results in a significant decrease in the sensing time theoretically by a factor of $\left(N_{c}+N_{d}\right)$. This is a very interesting property when the primary user becomes active and reclaims its band, since in such a case a small reaction time is necessary to reduce the disturbance caused to the primary user when it began to transmit.

\section{Simulation results}

In this section, we investigate by Monte-Carlo simulation the performance of the proposed CS-based GLRT detector in terms of $P_{d}$ versus $S N R$ of the primary user. To this end, we first 
discuss how the prior information about the noise power (i.e. the noise power uncertainty) affects the performance of the energy detector spectrum sensing algorithm. It is worth noting that it provides an upper bound for the spectrum sensing detection performance over the other detectors when the receiver knows the noise power and it is near optimal as shown in [25]. Then, we compare the performance of the proposed CS-based GLRT detector to the energy detector (ED), the sliding window (SW) detector and Axell detector (Ax) for some realistic scenarios. The wireless channel is assumed to be an additive white Gaussian noise (AWGN) channel. For each secondary user, the sensing period is assumed to contain $K$ OFDM blocks such that $L=K\left(N_{d}+\right.$ $\left.N_{c}\right)+N_{d}$ samples are used in each sensing algorithm. In all simulations, the transmitter sends an OFDM signal modulated with 16-QAM with $N_{d}$ subcarriers and a CP length of $N_{c}$, and the results are averaged over 1000 realizations.

The $P_{d}$ versus $S N R^{1}$ of the proposed detector is evaluated while keeping constant $P_{f a}$. A design probability of false alarm $P_{f a}=0.05$ is used to find the threshold multiplier $\eta$. Since we are interested by the low $S N R$ region, it is necessary to sense the primary user signal around the cell boundaries where the $S N R$ of the primary user is low, so it is varied from $-25 \mathrm{~dB}$ to $0 \mathrm{~dB}$. The threshold multiplier was determined numerically because we could not obtain a closed form expression for the $P_{f a}$. In Fig. 6, we present the performance of the ED spectrum sensing algorithm with known noise power $\sigma_{n}^{2}$ in the case of noise uncertainty (NU). The presence of thermal noise and noise due to transmissions by other users make the knowledge about the noise power impossible. Noise uncertainty is always present and there is at least about $1-2 d B$ in a real environment [27]. In the simulations, " $E D-\alpha d B$ " indicates that the energy detection has $\alpha d B$ of noise uncertainty.

\footnotetext{
${ }^{1}$ The SNR in dB is defined as $10 \log _{10}\left(\sigma_{s}^{2} / \sigma_{n}^{2}\right)$.
} 


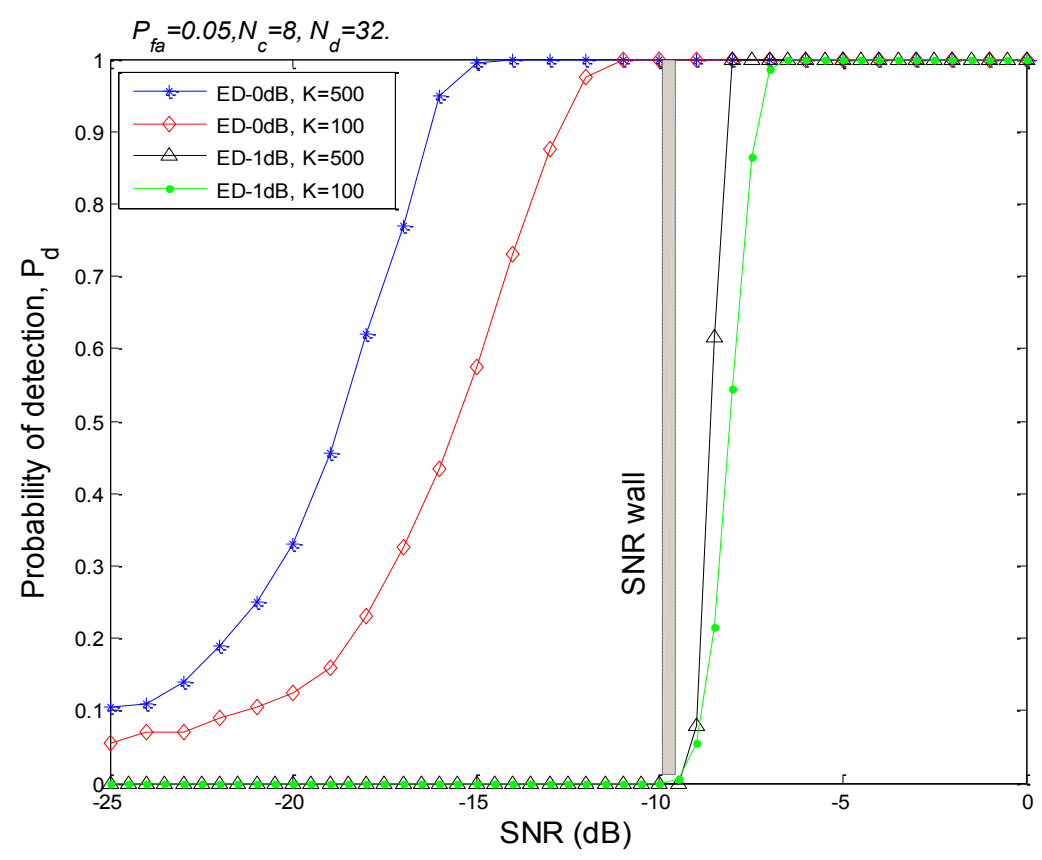

Fig. 6. Probability of detection versus SNR of the energy detector with and without noise power uncertainty.

In theory, the degradation in the detection performance due to reduced $S N R$ can be countered by increasing the detection time, as shown in Fig. 6, but unfortunately in practice there is a limit referred to as "SNR wall" below which the primary OFDM signal cannot be detected reliably, no matter how long the sensing time is. In fact, increasing the observation window does not add any significant improvement to the detection performance in the region of very low $S N R$. Hence, noise uncertainty imposes fundamental limitations on the detection performance of the energy detector. The existence of such limit has been also verified experimentally in [34]. On the other hand, cognitive radio systems do need detectors that perform well in low SNR region, in order to avoid any interference to the primary users. To this end, we developed a detector that exploits the inherent characteristics of the primary signal to improve system performance.

In the second scenario, we analyze the performance of the proposed detector varying the sensing time. Sensing time (or detection time) has an important role in determining the performance and energy consumption of the detector. In order to obtain reliable information about the impact of the observation window length $L=K\left(N_{c}+N_{d}\right)+N_{d}$ on the performance, 
we present in Fig. 7 the detection probability with different size of the observation window or equivalently with different values of $K$ as a function of the $S N R$. The size of the IFFT is set to 32 and thus $N_{d}=32$, the cyclic prefix CP is $N_{c}=N_{d} / 4=8$, representing $25 \%$ of the symbol duration before introducing $\mathrm{CP}$, and $K$ is changed from 200 to 800 . We observe that the detector sensitivity improves as the number of OFDM symbols increases; in other words, longer detection time implies better the detection performance. We note that a compromise has to be made between the detection performance and the sensing time, since the opportunistic transmission must be interrupted quickly when the primary user needs to use its frequency band. In addition, the detection time has direct impact on power consumption, especially when the amount of data that needs to be processed is high. Therefore, the detection time is an important factor to be taken into account to optimize the detection performance and the power consumption of the algorithm.

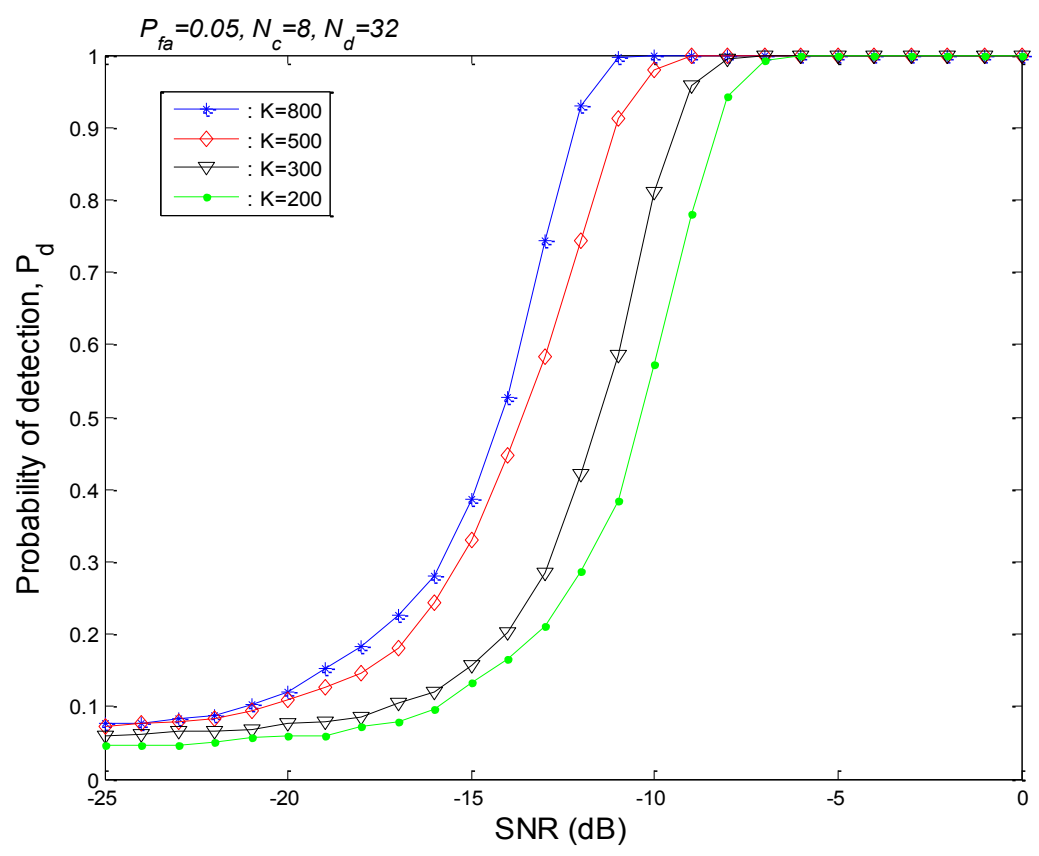

Fig. 7. Probability of detection versus SNR of the proposed detector for different detection time.

A comparison of the detection performance of the state of the art detectors described in Sect. III is provided in Fig. 8. The performance of the ED that knows $\sigma_{n}^{2}$ are also plotted as a benchmark of the detection performance. No detection performance improvement can be 
achieved over this clairvoyant ED [25]. From the obtained results, we also observe that the proposed detector outperforms Axell's detector. For example, in the IEEE 802.22 WRAN standard, a primary user DVB-T signal must be detected by a secondary user with $P_{d}>0.9$ [35]. For $P_{d}=0.9$, the performance improvement is on the order of $0.84 d B$. The $\mathrm{SW}$ detector is shown to perform better than the proposed scheme, but the SW detector requires knowledge of the exact value of the noise power $\sigma_{n}^{2}$, and thus its performance is strongly affected by the noise uncertainty, as will be shown in the following.

From Fig. 8, we observe that when the number of OFDM symbols increases, the detection performance of the proposed detector approaches to that of the SW detector, which assumes exact knowledge of $\sigma_{n}^{2}$, i.e. if the number of received OFDM symbols $K \rightarrow \infty$, these two detectors have the same performance. We also note that considering $K=400$ in the proposed sensing algorithm yields the same performance as Axell's detector with $K=500$.

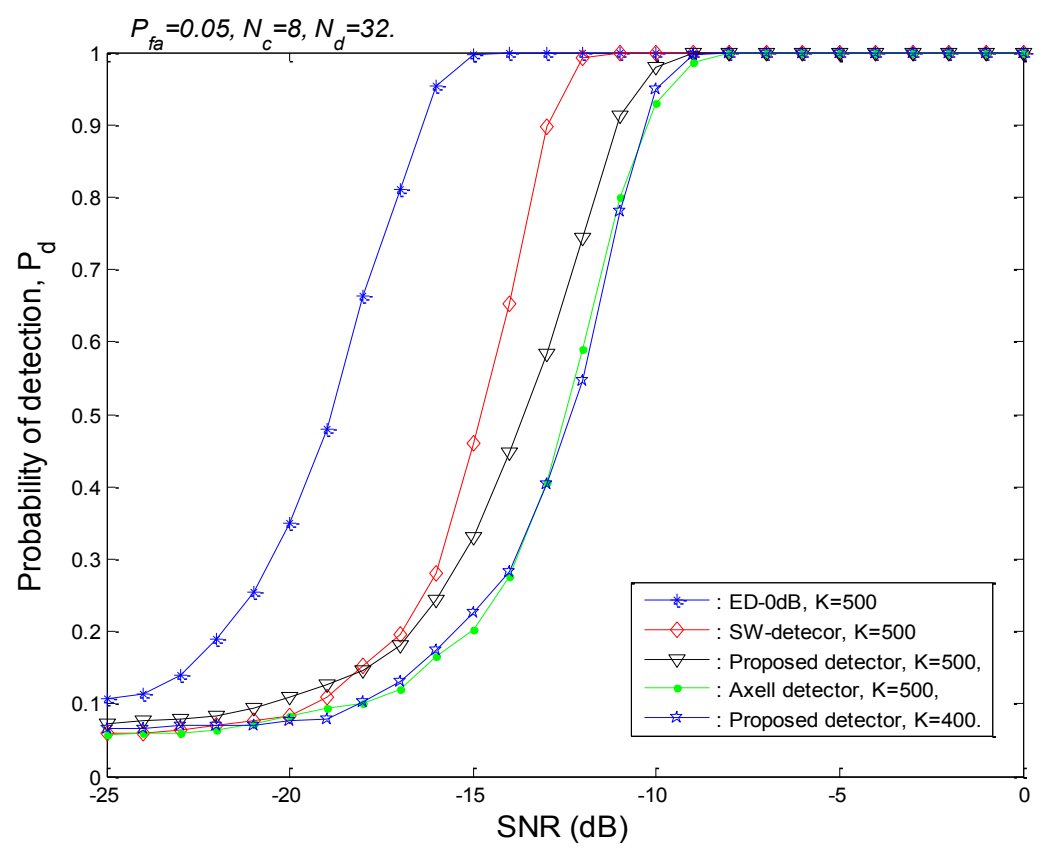

Fig. 8. Probability of detection versus SNR for various sensing algorithms.

In practice, the noise power is never perfectly known. Moreover, in the presence of interference, which could come from other secondary users or is caused by another operating electronic device in the vicinity, the resulting total noise power $\sigma_{n}^{2}$ is not known or is time varying. In the 
simulations presented in Fig. 9, we evaluate the impact of such situation on the detector performance by considering a $2 d B$ of noise uncertainty (i.e. the noise power deviate $2 \mathrm{~dB}$ from the true value). We observe that the proposed detector is not only robust but it is also able to adapt to the radio channel conditions. We also note that Axell's detector is robust against the noise power variation. On the other hand, the probability of detection of the ED is seriously degraded by a small noise uncertainty while the sliding window detector seems to be less sensitive. In conclusion, we see that the proposed detector is an adaptive threshold detector that outperforms all the other detectors considered in the presence of a small noise uncertainty.

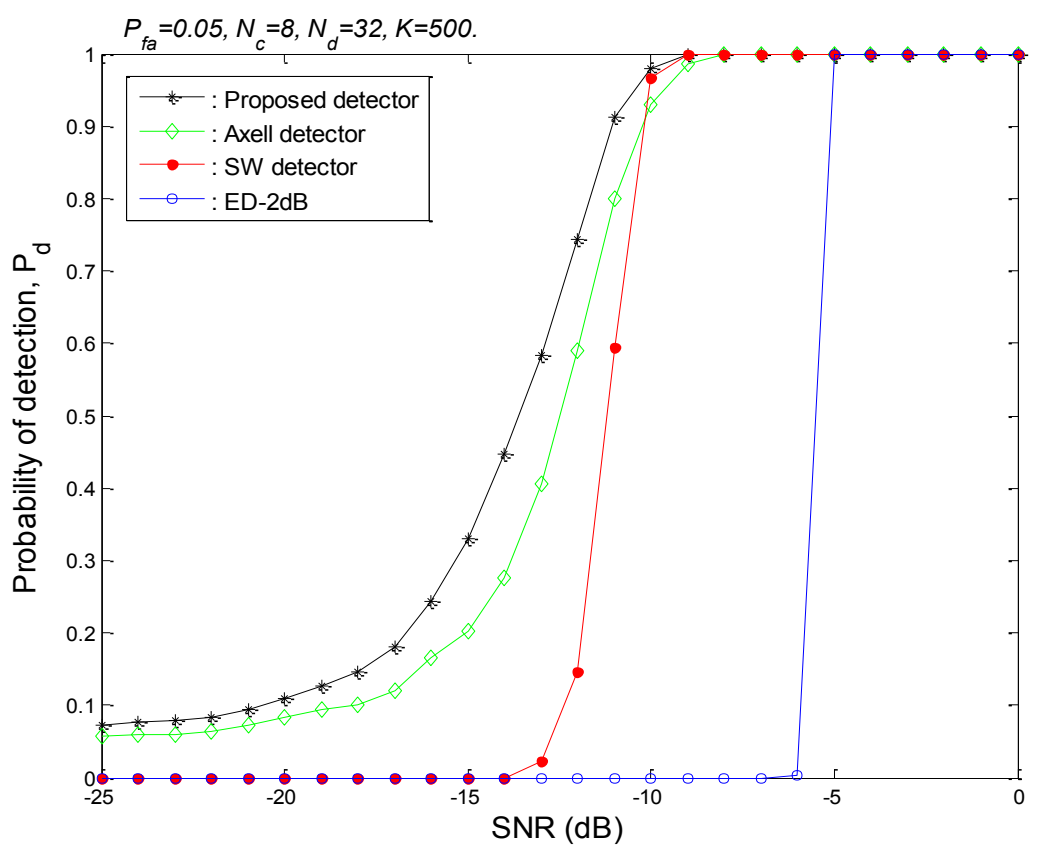

Fig. 9. Probability of detection versus SNR for various sensing algorithms with $2 \mathrm{~dB}$ of noise uncertainty.

We also investigated the effect of cyclic prefix (CP) length $N_{c}$ and of the number $N_{d}$ of subcarriers on the detection performance of the proposed detector. The primary signal is an OFDM based signal and, in order to maintain the same observation window length, we choose a sensing time corresponding to 20,000 OFDM signal samples, the CP length $N_{c}$ is varied from 1 to the maximum value $N_{d}$, and $N_{d}$ takes values from 16 to 128 . We get from Fig. 10 that the CP length plays a relevant role on the performance. Moreover, increasing $N_{c}$ the performance of the 
detector improves considerably, due to the stronger values of the correlation in the redundant data of the cyclic prefix. The best performance is obtained when $N_{c}=N_{d}$, i.e. when the cyclic prefix has the same length as the data length. This case is however not realistic, but it provides an idea of how much the CP length of the OFDM signals can improve the detection performance when then noise power is unknown relative to that of the energy detector ED-0dB when the noise power is perfectly known. In reality, just only little set of values for $N_{c}$ are used, for exemple DVB-T standard $N_{c}$ has only four values.

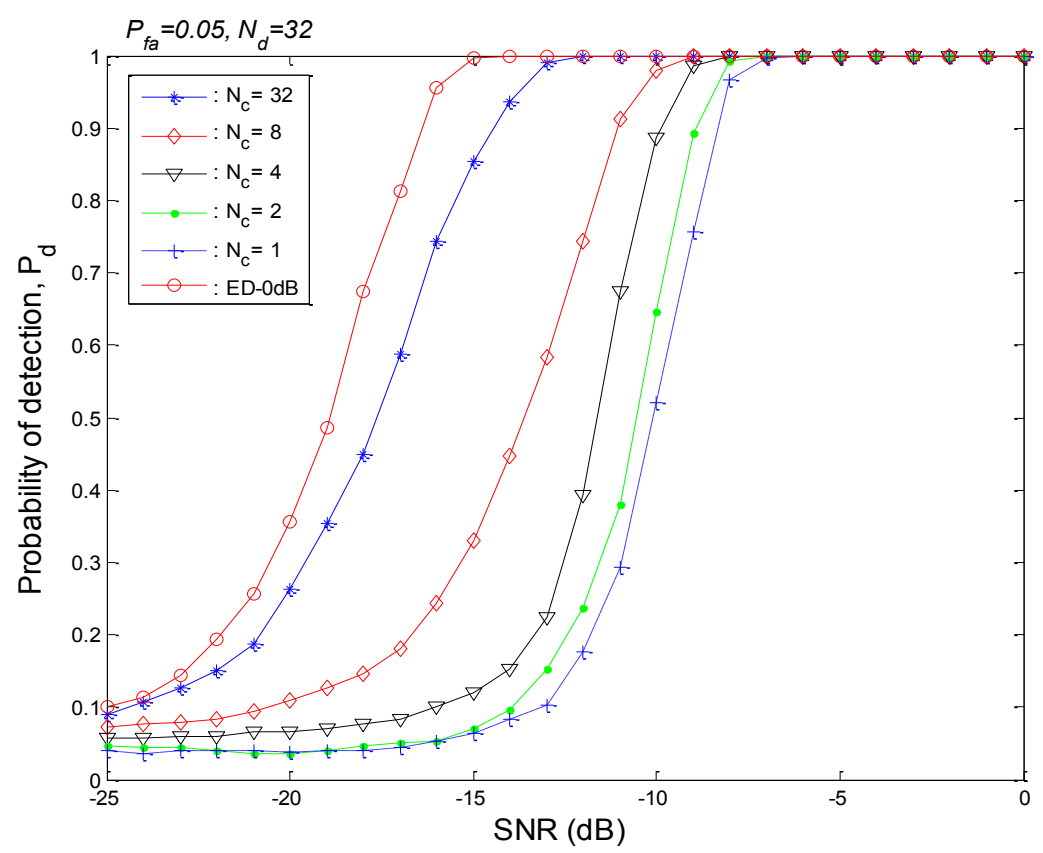

Fig. 10. Probability of detection versus SNR of the proposed detector for different values of the cyclic prefix $\mathrm{CP}$, with a sensing time of 20,000 samples.

In Fig. 11, we plot the detection performance of the CS-based GLRT detector for different values of the number $N_{d}$ of subcarriers. We observe that the larger $N_{d}$ is and the worse the performance of the detector are. The interpretation of the obtained result is increasing $N_{d}$ for a given $N_{c}$ is equivalent to decreasing $N_{c}$ for a given $N_{d}$ (less correlated samples in CP region as shown in figure 2) which reduces the probability of detection. On the contrary, the larger $N_{c}$ is and the better the performance are. These two observations about $N_{c}$ and $N_{d}$ suggest that the detection performance could be dependent only on the ratio $N_{c} / N_{d}$. 


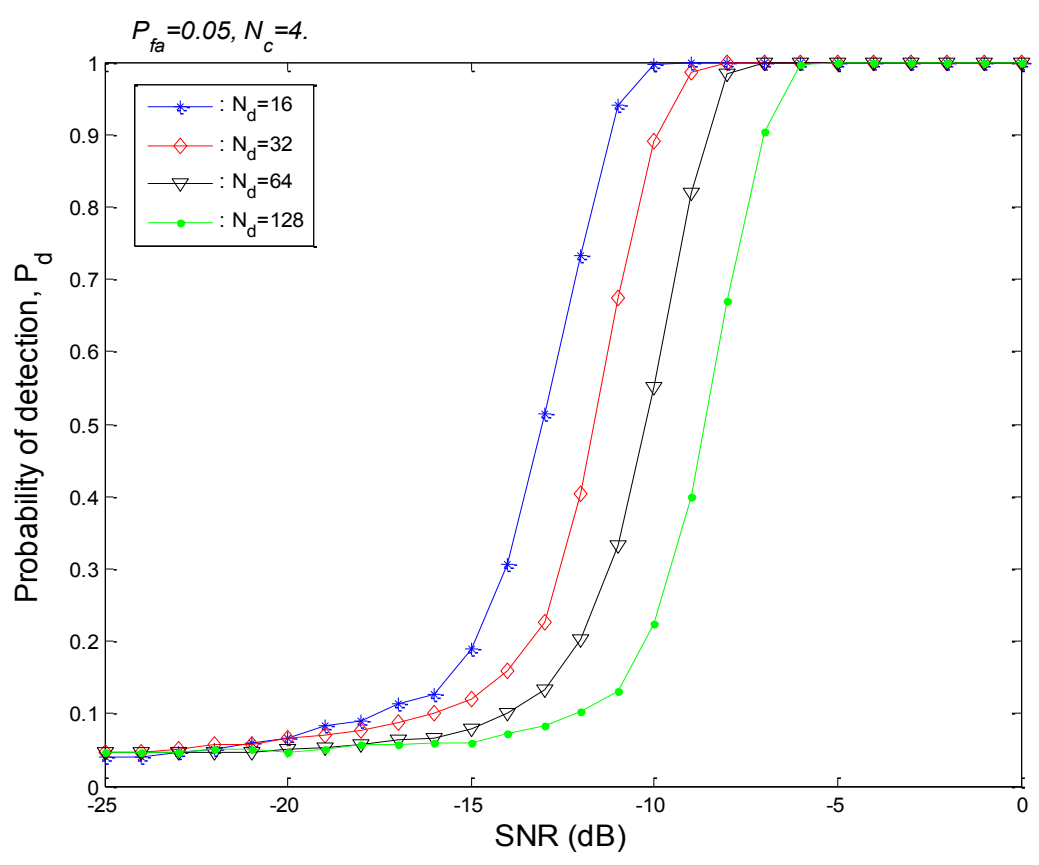

Fig. 11. Probability of detection versus SNR of the proposed detector for different time delay $\boldsymbol{N}_{\boldsymbol{d}}$ and a detection time of 20,000 samples.

To validate this hypothesis, we plot in Fig. 12 the detection performance for three different wireless standards, the WLAN, DVB-T2 and LTE based on different CP lengths. The standards are summarized as [36]:

- WLAN: $N_{d}=52, N_{c}=13$, number of OFDM symbols $K=462$ thus an observation window length $L=462\left(N_{d}+N_{c}\right)$ and $\left(N_{c} / N_{d}=1 / 4\right)$,

- DVB-T2 1k mode: $N_{d}=1024, N_{c}=128$, number of OFDM symbols $K=26$ and observation window length $L=26\left(N_{d}+N_{c}\right)$ and $\left(N_{c} / N_{d}=1 / 8\right)$,

- LTE: $N_{d}=512, N_{c}=36$, number of OFDM symbols $K=55$ and observation window length $L=55\left(N_{d}+N_{c}\right)$ and $\left(N_{c} / N_{d}=0.07\right)$.

We have considered the same detection time corresponding to 30,000 samples of OFDM signal with the corresponding parameters $N_{c}$ and $N_{d}$ as shown above. We observe an enhancement of the detection performance when the ratio $N_{c} / N_{d}$ for the corresponding OFDM based wireless standard increases, which confirms the aforementioned conclusion. 


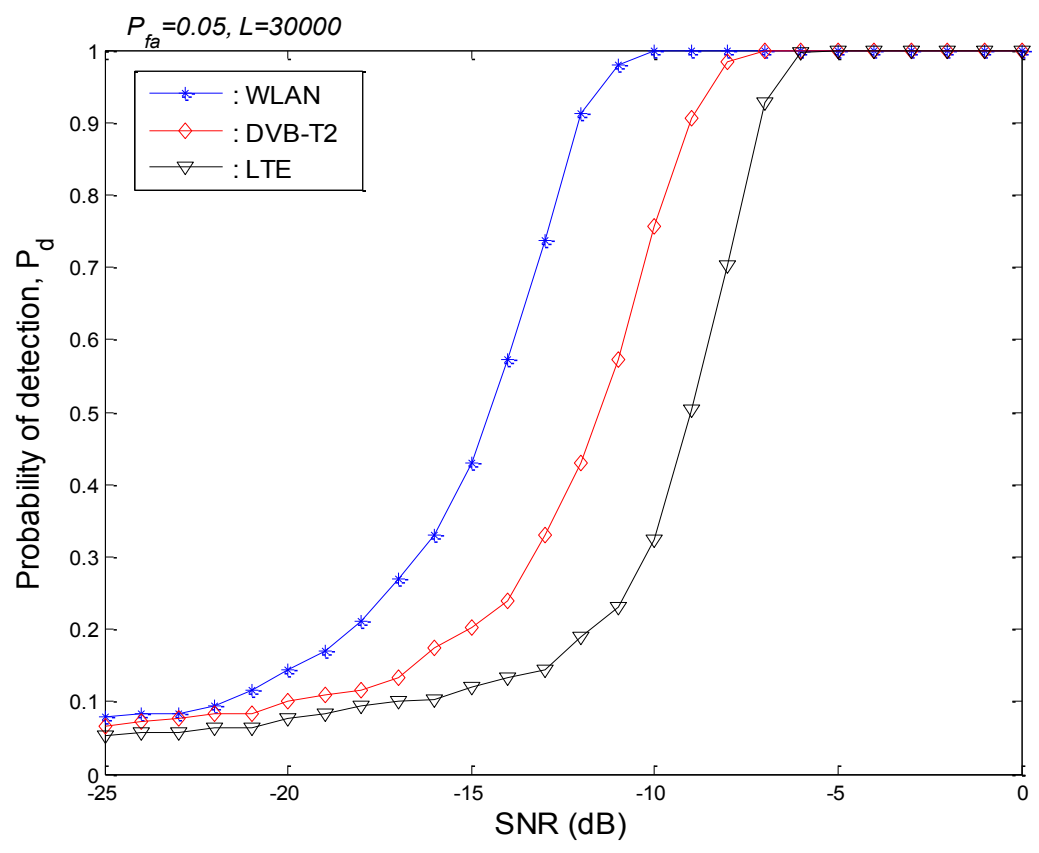

Fig. 12. Probability of detection versus SNR of the proposed detector, for three wireless standards; observation window size $=30,000$ OFDM samples.

\section{Conclusions}

In this paper, we have proposed a new fast spectrum sensing algorithm for the detection of unsynchronized OFDM signals embedded in additive white Gaussian noise. A CS-based spectrum sensing algorithm was derived that is computationally efficient by exploiting the data cyclostationarity, i.e. the periodicity of the data autocorrelation function, induced by the presence of the cyclic prefix $\mathrm{CP}$ in the data. The algorithm makes use of a sample estimate of the noise power to set an adaptive threshold, in order to maintain constant probability of false alarm in the presence of background noise fluctuations, due to varying thermal noise or interferences from other users.

The performance of the proposed detector were investigated by Monte Carlo simulation using OFDM signals under various test scenarios, and compared to those of the energy detector, the sliding window detector, and Axell's detector. The obtained results show an improvement in detection performance at low SNR, a significant reduction in complexity and a short sensing time of the proposed algorithm. This last property is of great interest for the implementation of the algorithm in cognitive radio systems. Moreover, contrary to the energy detector, the proposed 
algorithm is robust against noise fluctuation and is superior to the method proposed in [25]. To conclude, we expect that the proposed spectrum sensing scheme can be considered as good candidate for practical implementation of OFDM-based cognitive radio systems attempting to improve the efficiency of radio spectrum usage especially in time varying background noise.

\section{Acknowledgment}

The authors would like to thank the reviewers for their valuable and constructive comments which certainly led to the enhancement of the quality of the paper.

\section{References}

[1] FCC, "Spectrum policy task force report," Tech. Rep. 02-135, Federal Communications Commission, November 2002, Available: http://hraunfoss.fcc.gov/edocs public/attachmatch/DOC-228542A1.pdf.

[2] M.A. McHenry, "NSF spectrum occupancy measurements project summary," Tech. Rep., SSC, August 2005, Available: http://www.sharedspectrum.com/.

[3] S. Haykin, "Cognitive radio: brain-empowered wireless communications," IEEE Journal on Selected Areas in Communications, vol. 23, no. 2, pp. 201-220, February 2005.

[4] J. Mitola and G. Q. Maguire, "Cognitive Radio: Making Software Radios More Personal," IEEE Personal Communications, vol. 6, no. 4, pp. 13-18, August 1999.

[5] J. Mitola, "Cognitive radio: An integrated agent architecture for software defined radio," Ph.D. dissertation, KTH Royal Institute of Technology, Stockholm, Sweden, 2000.

[6] E. Hossain and V. K. Bhargava, Cognitive Wireless Communication Networks, Springer US, 2007.

[7] J. Ma, G. Y. Li, and B. H. Juang, "Signal processing in cognitive radio," Proceedings of the IEEE, vol. 97, no. 5, pp. 805-823, May 2009.

[8] E. Larsson and M. Skoglund, "Cognitive radio in a frequency-planned environment: some basic limits," IEEE Transactions on Wireless Communications, vol. 7, no. 12, pp. 48004806, December 2008.

[9] H. Tang, "Some Physical Layer Issues of Wide-band Cognitive Radio Systems," in Proceedings of IEEE Symposium on New Frontiers in Dynamic Spectrum Access Networks, pp. 151-159, November 2005.

[10] T. Yücek and H. Arslan, "A Survey of Spectrum Sensing Algorithms for Cognitive Radio Applications", IEEE Communications Survey and Tutorials, Vol.11, no.1, First Quarter 2009. 
[11] A. Ghasemi and E. S. Sousa, "Spectrum sensing in cognitive radio networks: requirements, challenges and design trade-offs," IEEE Communications Magazine, vol. 46, no. 4, pp. 32 39, April 2008.

[12] S. Haykin, D. J. Thomson, and J. H. Reed, "Spectrum sensing for cognitive radio," Proceeding IEEE, vol. 97, no. 5, pp. 849-877, May 2009.

[13] C.-H. Hwang, G.-L. Lai, and S.-C. Chen, "Spectrum-sensing in wideband OFDM cognitive radios," IEEE Transaction on Signal Processing, vol. 58, no. 2, pp. 709-719, February 2010 .

[14] R. Tandra and A. Sahai, "SNR walls for signal detection," IEEE Journal of Selected Topics in Signal Processing, vol. 2, no. 1, pp. 4-17, February 2008.

[15] A. V. Dandawaté and G. B. Giannakis, "Statistical tests for presence of cyclostationarity," IEEE Transactions on Signal Processing, vol. 42, no. 9, pp. 2355-2369, September 1994.

[16] J. Lundén, V. Koivunen, A. Huttunen, and H. V. Poor, "Collaborative cyclostationary spectrum sensing for cognitive radio systems," IEEE Transactions on Signal Processing, vol. 57, no. 11, pp. 4182-4195, November 2009.

[17] P. Sutton, K. Nolan, and L. Doyle, "Cyclostationary signatures in practical cognitive radio applications," IEEE Journal on Selected Areas in Communications, vol. 26, no. 1, pp. 1324, January 2008.

[18] T. E. Bogale and L. Vandendorpe, "Multi-cycle cyclostationary based spectrum sensing algorithm for OFDM signals with noise uncertainty in cognitive radio networks," MILITARY COMMUNICATIONS CONFERENCE, 2012 - MILCOM, 2012, pp. 1-6.

[19] Sohn Sung Hwan, Han Ning, Jae Moung Kim, and Jae Wan Kim, "OFDM Signal Sensing Method Based on Cyclostationary Detection," 2nd International Conference on Cognitive Radio Oriented Wireless Networks and Communications, CrownCom 2007, August 2007, pp. 63-68.

[20] K. Berbra, M. Barkat, and A. Anou, "PN Code Acquisition Using Smart Antenna and Adaptive Thresholding CFAR Based on Ordered Data Variability for CDMA Communications," Progress in Electromagnetics Research B, vol. 57, pp. 139-155, January 2014.

[21] A. Sofwan, M. Barkat, and S.A. AlQahtani, "PN code acquisition using smart antennas and adaptive thresholding for spread spectrum communications," The Journal of Mobile Communication, Computation and Information, vol. 2, no. 1, pp. 1-12, May 2015.

[22] S. Chaudhari, V. Koivunen, and H. V. Poor, "Autocorrelation-Based Decentralized Sequential Detection of OFDM Signals in Cognitive Radios", IEEE Transactions on Signal Processing, vol. 57, no. 7, pp. 2690-2700, July 2009.

[23] L. Zhongding and F. Chin, "Sensing OFDM Systems Under Frequency-Selective Fading Channels," IEEE Transactions on Vehicular Technology, vol. 59, no. 4, pp. 1960-1968, May 2010. 
[24] Huawei Technologies and UESTC, "Sensing scheme for DVB-T," IEEE Std.802.2206/0127r1, July 2006.

[25] E. Axell and E. G. Larsson, "Optimal and Sub-Optimal Spectrum Sensing of OFDM Signals in Known and Unknown Noise Variance," IEEE Journal on Selected Areas in Communications, vol. 29, no. 2, pp. 290-304, February 2011.

[26] S. Bokharaiee, H. Nguyen, and E. Shwedyk, "Blind spectrum sensing for OFDM-based cognitive radio systems," IEEE Transactions on Vehicular Technology, vol. 60, no. 3, pp. 858-871, March 2011.

[27] R. Tandra and A. Sahai, "Fundamental limits on detection in low SNR under noise uncertainty," IEEE International Conference on Wireless Networks, Communications and Mobile Computing, June 13-16 2005, vol. 1, pp. 464-469.

[28] M. Barkat and P. K. Varshney, "Decentralized CFAR detection," IEEE Transactions on Aerospace and Electronic Systems, vol. 25, no. 2, pp. 141-149, March 1989.

[29] M. Barkat, Signal detection and estimation, 2nd ed. Boston, MA, Artech House, 2005.

[30] M. Greco, F. Gini and M. Diani, "Robust CFAR detection of random signals in compoundGaussian clutter plus thermal noise," IEE proceedings Radar, Sonar and Navigation, vol. 148, no. 4, pp. 227-232, August 2001.

[31] Q. Zhao and B. M. Sadler, "A Survey of Dynamic Spectrum Access: Signal Processing, Networking, and Regulatory Policy,” IEEE Signal Processing Magazine, vol. 24, no. 3, pp. 79-89, May 2007.

[32] H. Urkowitz, "Energy detection of unknown deterministic signals," Proceedings of the IEEE, vol. 55, no. 4, pp. 523-531, April 1967.

[33] D. Danev, E. Axell, E. D. Larsson, "Spectrum sensing methods for detection of DVB-T signals in AWGN and fading channels," in 2010 IEEE 21st International Symposium on Personal Indoor and Mobile Radio Communications (PIMRC), September 26-30 2010, pp. 772-776.

[34] D. Cabric, A. Tkachenko, and R. W. Brodersen, "Experimental study of spectrum sensing based on energy detection and network cooperation," in Proceedings of the First International Workshop on Technology and Policy for Accessing Spectrum (TAPAS 2006), August 2006, pp. 1-8.

[35] S. Shellhammer and G. Chouinard, "Spectrum sensing requirements summary," IEEE Std.802.22-06/0089r4, June 2006.

[36] S. Chaudhari, J. Lunden, and V. Koivunen, "Collaborative autocorrelation-based spectrum sensing of ofdm signals in cognitive radios," in Information Sciences and Systems, 2008. CISS 2008. 42nd Annual Conference on, March 19-21 2008, pp. 191-196. 